\title{
A dual cam system for four-bar motion generation with adjustable length crank and follower links
}

\author{
Wen-Tzong LEE*, Yahia M. AL-SMADI** and Kevin RUSSELL*** \\ ${ }^{*}$ Department of Biomechatronics Engineering, National Pingtung University of Science and Technology \\ 1, Shuefu Road, Neipu, Pingtung 91201, Taiwan \\ E-mail: wtlee@npust.edu.tw \\ ${ }^{* *}$ Department of Mechanical Engineering, Jordan University of Science and Technology \\ Ar Ramtha 3030، Ar-Ramtha, Jordan \\ *** Department of Mechanical and Industrial Engineering, New Jersey Institute of Technology \\ University Heights, Newark, New Jersey 07102, USA
}

Received: 28 March 2021; Revised: 5 August 2021; Accepted: 9 September 2021

\begin{abstract}
The objective in planar four-bar motion generation is to calculate the linkage dimensions required to achieve a group of prescribed coupler positions. For a given planar four-bar motion generator however, the rolling motion of its moving centrode over its fixed centrode will replicate its coupler motion. The curvature of the fixed and moving centrodes can be incorporated as contact surfaces in a rolling cam system to replicate the coupler motion of a planar four-bar motion generator. While the advantages that cam-based systems have over four-bar linkage-based systems are application-specific, some of the advantages relate to system workspace (having a more compact design), structural soundness (having a greater load bearing capacity) and design simplicity (utilizing fewer components). While design methods for cam-based systems have been developed for conventional non-adjustable four-bar motion generators, no such method has been presented for the adjustable planar four-bar motion generator. Given the design advantages associated with cam-based systems along with an absence of published work in the design of cam-based systems for adjustable motion generation, an opportunity exists to contribute to the body of knowledge in this area. This work presents for the first time a design method for a dual cam system to replicate the coupler motion of an adjustable planar four-bar motion generator. This design method incorporates an optimization model for defect-free adjustable planar four-bar motion generation. The adjustments considered in the optimization model are adjustable crank and follower moving pivots with adjustable crank and follower link lengths. This design method also incorporates centrode generation equations for the adjustable planar four-bar motion generator. As an example, the centrodes generated for a calculated adjustable motion generator were incorporated into a computer-aided design model to produce a concept dual cam system.
\end{abstract}

Keywords : Motion generation, Centrode generation, Planar linkage, Four-bar linkage, Adjustable linkage, Cam design

\section{Introduction}

\subsection{Adjustable planar four-bar motion generation}

The objective in four-bar motion generation is to calculate the linkage dimensions to achieve a single group of prescribed coupler positions. In 2018, a survey work was produced on the developments made in quantitative four-bar motion generation from 1970-2018 (Lee and Russell, 2018).

A single four-bar motion generator achieves a single group of prescribed coupler positions. If a four-bar linkage can be designed with dimensions that can be adjusted, a single linkage can achieve multiple groups of prescribed coupler positions. Figure 1 includes the moving pivot (the non-grounded revolute joint) adjustment options for crank and follower links. In this work, the adjustable length moving pivot adjustment is considered over the constant length moving pivot adjustment since the former offers more moving pivot adjustments. Adjustability gives a four-bar motion 
generator a greater range of flexibility in terms of the number of prescribed position groups the linkage can achieve. Subsequently, the objective in adjustable four-bar motion generation is to calculate the dimensions of an adjustable linkage to achieve multiple groups of prescribed coupler positions.

The same 2018 survey work include developments made in quantitative adjustable four-bar motion generation (Lee and Russell, 2018). Four classes of motion generation problems were defined and solved for a planar four bar linkage having adjustable driven crank pivots (Ahmad and Waldron,1979). The classes are the number of position combinations achieved with five positions expressed in two groups (e.g., $\mathrm{P}_{1}-\mathrm{P}_{2}-\mathrm{P}_{3}: \mathrm{P}_{1}-\mathrm{P}_{4}-\mathrm{P}_{5}$ ). A Method of Least Squares-based motion generation model (for prescribing two groups of expanded positions) was presented for an adjustable spherical four-bar linkage (Lee et al., 2009). This model considers constant length crank and follower link moving pivot adjustments. Motion generation models for fixed length and adjustable length moving pivot adjustments for the R-R and S-S dyads were presented for the spatial four-bar RRSS linkage (Russell and Sodhi, 2001). These models were demonstrated in calculating adjustable RRSS motion generators to approximate two and three groups of prescribed positions. The adjustable RRSS motion generation models were also expanded to incorporate position tolerances to produce corresponding solution regions from which R-R dyad solutions are chosen for adjustable spatial RRSS and adjustable planar four-bar motion generation (Russell and Sodhi, 2002, Russell and Sodhi, 2004). Finite and multiply separated position motion generation models for fixed length moving pivot adjustable R-R and C-S dyads were presented for the spatial four-bar RRSC linkage (Russell and Sodhi, 2001). These models were demonstrated in calculating adjustable RRSC motion generators to approximate two groups of prescribed positions, velocities, and accelerations. A constrained optimization model for defect-free motion generation was presented for fixed length moving pivot adjustable planar four-bar linkages (Shen, 2015). This model was demonstrated in calculating an adjustable motion generator that was also order, branch and circuit defect free. By equipping a single planar five-bar linkage with joints that can be constrained during linkage motion, four unique four-bar linkage configurations can be achieved for adjustable motion generation (Venkataramanujam and Larochelle, 2016). This method introduced a class of mechanical devices called reconfigurable motion generators. Fixed length moving pivot adjustments for the planar four-bar linkage were considered for three groups (also called phases) of prescribed positions (Wang and Sodhi, 1996). Incorporating an actuator as the coupler in the design of a planar four-bar linkage allows for fixed length moving pivot adjustments (Wu and Chen, 2005). Rather than permitting a fixed adjustment over linkage motion, the actuator allows continuous adjustment over linkage motion. Follower link fixed length fixed pivot adjustments were also considered in adjustable planar four-bar motion generation (Zhou and Cheung, 2004). In this work, a modified genetic algorithm was employed to seek the global optimal solution.

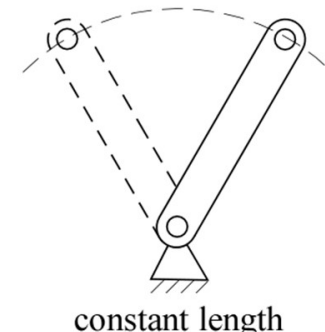
moving pivot adjustment

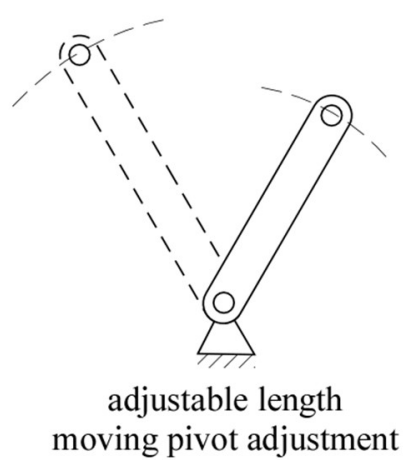

Fig. 1 Moving pivot adjustment options for crank and follower links.

\subsection{Centrode generation and cam design}

At any instant in time during linkage motion, the coupler of a planar four-bar linkage undergoes pure rotation about an imaginary rotation center called an instant center of velocity (Russell et al., 2019). The planar four-bar linkage has 6 unique instant centers according to the instant center equation $\mathrm{N}_{\mathrm{IC}}=n(n-1) / 2$ where $\mathrm{N}_{\mathrm{IC}}$ is the total number of instant centers and $n$ is the total number of links. The instant center of focus in this work is the point of intersection between 
lines passing through the crank and follower links (point $\mathbf{X}$ in Figure 2).

Over the motion of the linkage, a locus of instant centers (called a centrode) is produced. The fixed centrode is produced over the motion of a four-bar linkage and the moving centrode is produced over the motion of the linkage where the coupler and ground links are inverted. The rolling motion of the moving centrode over the fixed centrode of a given four-bar linkage perfectly replicates its coupler motion (Russell et al., 2019). Centrodes can be used to design cam or gear systems that replicate the desired coupler motion of a planar four-bar linkage (Lee et al., 2020).

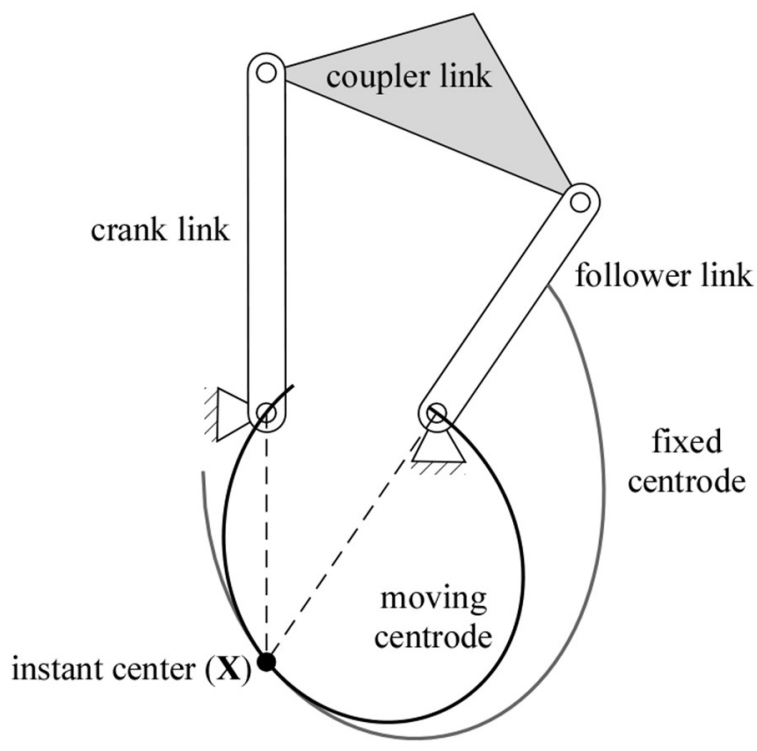

Fig. 2 Planar four-bar linkage with fixed and moving centrodes.

\subsection{Objective and scope of work}

The objective of this work is to present and demonstrate a dual cam system design method to replicate the coupler motion of an adjustable planar four-bar linkage. The adjustable linkage considered is a planar-four bar linkage with adjustable crank and follower lengths and moving pivots. Works have been published relating to adjustable motion generation (Section 1.1) and centrode generation for the design of cam systems for motion generation (Section 1.2). However, no work has been published to date that considers the design of cam systems for adjustable motion generation. Therefore, this work combines both adjustable planar four-bar motion generation and planar four-bar centrode generation to produce a design method for cam systems to replicate adjustable planar four-bar motion generators.

\section{Optimization model for adjustable planar four-bar motion generation}

Figure 3 includes a planar four-bar linkage with adjustable crank and follower length moving pivot adjustments. Also included in this figure are the linkage dimensions and angular displacement variables. In this figure, the variable $\theta$ is the angular displacement of the crank link (link $\mathbf{a}_{0}-\mathbf{a}_{1}$ or $\mathbf{a}_{0}-\mathbf{a}_{1 n}$ ) about $\mathbf{a}_{0}$. In Figure 3, the variable $\alpha$ is the angular displacement of the coupler link (about $\mathbf{a}_{1}$ or $\mathbf{a}_{1 n}$ ). These displacement angles are measured with respect to the initial positions of the crank and follower links respectively. The crank and follower fixed pivots are $\mathbf{a}_{0}$ and $\mathbf{b}_{0}$ respectively. The crank link moving pivot adjustments are $\mathbf{a}_{1}$ and $\mathbf{a}_{1 n}$. The follower link moving pivot adjustments are $\mathbf{b}_{1}$ and $\mathbf{b}_{1 n}$. The subscript $n$ in $\mathbf{a}_{1 n}$ and $\mathbf{b}_{1 n}$ is used to differentiate the adjusted moving pivots from the original moving pivots $\mathbf{a}_{1}$ and $\mathbf{b}_{1}$ (see Figure 3). The coupler dimensions $\mathbf{p}$ and $\mathbf{q}$ correspond to the coupler positions prescribed by the user while $\mathbf{p}^{*}$ and $\mathbf{q}^{*}$ correspond to those achieved by the calculated four-bar motion generator (see Figure 3).

A constrained optimization model was prepared to calculate the $\mathrm{x}$ and y-components of $\mathbf{a}_{0}, \mathbf{a}_{1}, \mathbf{a}_{1 n}, \mathbf{b}_{0}, \mathbf{b}_{1}$ and $\mathbf{b}_{1 n}$ as well as $\theta$ and $\alpha$ values for an adjustable planar four-bar linkage given two groups of five coupler positions (positions 1-2-3-4-5 and 1-6-7-8-9). In this optimization model, the objective function is 


$$
f(\mathbf{X})=\sum_{j=2}^{5}\left\{\left\|\mathbf{p}_{j}-\mathbf{p}_{j}^{*}\right\|^{2}+\left\|\mathbf{q}_{j}-\mathbf{q}_{j}^{*}\right\|^{2}\right\}+\sum_{k=6}^{9}\left\{\left\|\mathbf{p}_{k}-\mathbf{p}_{k}^{*}\right\|^{2}+\left\|\mathbf{q}_{k}-\mathbf{q}_{k}^{*}\right\|^{2}\right\}
$$

where $\mathbf{X}=\left(a_{0 x}, a_{0 y}, a_{1 x}, a_{1 y}, b_{0 x}, b_{0 y}, b_{1 x}, b_{1 y}, b_{1 n x}, b_{1 n y}, \theta_{2-9}, \alpha_{2-9}\right)$. In Equation (1), $\mathbf{p}_{j}$ and $\mathbf{q}_{j}$ as well as $\mathbf{p}_{k}$ and $\mathbf{q}_{k}$ are the user-prescribed coupler positions. In the same equation, $\mathbf{p}_{j}^{*}$ and $\mathbf{q}_{j}^{*}$ as well as $\mathbf{p}_{k}^{*}$ and $\mathbf{q}_{k}^{*}$ are the coupler positions achieved by the calculated adjustable four-bar motion generator. In the objective function, the error (the squared norm of the differences) between the prescribed and achieved coupler positions is minimized.

Equations (2) through (5) are the equality constraints in the optimization model (Suh and Radcliffe, 1978). Equations (2) and (3) maintain a constant crank length during linkage motion within each adjustment. Equations (4) and (5) maintain a constant follower length during linkage motion within each adjustment.

In addition to constant length equality constraints, the optimization model also includes inequality constraints to eliminate branch defects (Balli and Chand, 2002). A single planar four-bar linkage at any crank position has two assembly configurations (meaning two ways to position the coupler and follower links to assemble the linkage) known as branches. A branch defect is an undesired state in four-bar motion generation where the prescribed positions are not achieved over a single linkage configuration. The cross product of the vector passing through $\mathbf{b}_{1}\left(\right.$ or $\left.\mathbf{b}_{1 n}\right)$ and $\mathbf{b}_{0}$ and the vector passing through $\mathbf{a}_{1}$ (or $\mathbf{a}_{1 n}$ ) and $\mathbf{b}_{0}$ will be either positive (indicating one branch) or negative (indicating the other branch). The inclusion of an inequality for the cross product that constrains the result to either exclusively positive or negative values subsequently constrains the motion generator to single branch over each achieved coupler position. Inequality (6) eliminates branch defects during linkage motion in the first linkage adjustment and Inequality (7) does the same in the second adjustment.

Equations (8) through (15) are planar four-bar kinematic motion equations that are incorporated in the optimization model (Suh and Radcliffe, 1978). Equations (8) through (11) calculate the motion of moving pivots $\mathbf{a}_{1}, \mathbf{a}_{1 n}, \mathbf{b}_{1}$ and $\mathbf{b}_{1 n}$ respectively and are included in Equations (2) through (7). Equations (12) through (15) calculate the motion of coupler points $\mathbf{p}_{1}^{*}$ and $\mathbf{q}_{1}^{*}$ and are included in Equation (1). In Equations (8) through (15), matrices $\left[R_{\theta_{j}}\right]$ and $\left[R_{\alpha_{j}}\right]$ are $3 \times 3$ planar rotation matrices for displacement angles $\theta$ and $\alpha$ respectively (Figure 3 ). The $\mathbf{a}, \mathbf{b}, \mathbf{p}$ and $\mathbf{q}$ terms throughout Equations (1) through (15) are all 3x1 column vectors having components of $\mathrm{x}, \mathrm{y}$ and unity).

Lastly, the optimization model includes inequality constraints to eliminate order defects (Balli and Chand, 2002). This is another undesired state in four-bar motion generation where coupler positions are not achieved in their prescribed order. Inequality (16) ensures a continuous counterclockwise crank rotation (or clockwise by reversing the inequality signs) over the achieved coupler positions.

The adjustable four-bar motion generation optimization model was codified in the commercial mathematical analysis software Matlab and solved using the interior point algorithm with algebraic gradients and Hessian (Nocedal and Wright, 2006).

$$
\begin{aligned}
& \left(\mathbf{a}_{j}-\mathbf{a}_{0}\right)^{T}\left(\mathbf{a}_{j}-\mathbf{a}_{0}\right)-\left(\mathbf{a}_{1}-\mathbf{a}_{0}\right)^{T}\left(\mathbf{a}_{1}-\mathbf{a}_{0}\right)=0, j=2,3 \ldots 5 \\
& \left(\mathbf{a}_{n k}-\mathbf{a}_{0}\right)^{T}\left(\mathbf{a}_{n k}-\mathbf{a}_{0}\right)-\left(\mathbf{a}_{1 n}-\mathbf{a}_{0}\right)^{T}\left(\mathbf{a}_{1 n}-\mathbf{a}_{0}\right)=0, k=6,7 \ldots 9 \\
& \left(\mathbf{b}_{j}-\mathbf{b}_{0}\right)^{T}\left(\mathbf{b}_{j}-\mathbf{b}_{0}\right)-\left(\mathbf{b}_{1}-\mathbf{b}_{0}\right)^{T}\left(\mathbf{b}_{1}-\mathbf{b}_{0}\right)=0, j=2,3 \ldots 5 \\
& \left(\mathbf{b}_{n k}-\mathbf{b}_{0}\right)^{T}\left(\mathbf{b}_{n k}-\mathbf{b}_{0}\right)-\left(\mathbf{b}_{1 n}-\mathbf{b}_{0}\right)^{T}\left(\mathbf{b}_{1 n}-\mathbf{b}_{0}\right)=0, k=6,7 \ldots 9 \\
& {\left[\left(\mathbf{b}_{j}-\mathbf{b}_{0}\right) \times\left(\mathbf{a}_{j}-\mathbf{b}_{0}\right)\right] \cdot\left[\left(\mathbf{b}_{1}-\mathbf{b}_{0}\right) \times\left(\mathbf{a}_{1}-\mathbf{b}_{0}\right)\right]>0, j=2,3 \ldots 5} \\
& {\left[\left(\mathbf{b}_{n k}-\mathbf{b}_{0}\right) \times\left(\mathbf{a}_{n k}-\mathbf{b}_{0}\right)\right] \cdot\left[\left(\mathbf{b}_{1 n}-\mathbf{b}_{0}\right) \times\left(\mathbf{a}_{1 n}-\mathbf{b}_{0}\right)\right]>0, k=6,7 \ldots 9} \\
& \mathbf{a}_{j}=\left[R_{\theta_{j}}\right]\left(\mathbf{a}_{1}-\mathbf{a}_{0}\right)+\mathbf{a}_{0}, j=2,3 \ldots 5 \\
& \mathbf{a}_{n k}=\left[R_{\theta_{k}}\right]\left(\mathbf{a}_{1 n}-\mathbf{a}_{0}\right)+\mathbf{a}_{0}, k=6,7 \ldots 9
\end{aligned}
$$




$$
\begin{aligned}
& \mathbf{b}_{j}=\left[R_{\alpha_{j}}\right]\left(\left[R_{\theta_{j}}\right]\left(\mathbf{b}_{1}-\mathbf{a}_{0}\right)+\mathbf{a}_{0}-\mathbf{a}_{j}\right)+\mathbf{a}_{j}, j=2,3 \ldots 5 \\
& \mathbf{b}_{n k}=\left[R_{\alpha_{k}}\right]\left(\left[R_{\theta_{k}}\right]\left(\mathbf{b}_{1 n}-\mathbf{a}_{0}\right)+\mathbf{a}_{0}-\mathbf{a}_{k}\right)+\mathbf{a}_{k}, k=6,7 \ldots 9 \\
& \mathbf{p}_{j}^{*}=\left[R_{\alpha_{j}}\right]\left(\left[R_{\theta_{j}}\right]\left(\mathbf{p}_{1}^{*}-\mathbf{a}_{0}\right)+\mathbf{a}_{0}-\mathbf{a}_{j}\right)+\mathbf{a}_{j}, j=2,3 \ldots 5 \\
& \mathbf{q}_{j}^{*}=\left[R_{\alpha_{j}}\right]\left(\left[R_{\theta_{j}}\right]\left(\mathbf{q}_{1}^{*}-\mathbf{a}_{0}\right)+\mathbf{a}_{0}-\mathbf{a}_{j}\right)+\mathbf{a}_{j}, j=2,3 \ldots 5 \\
& \mathbf{p}_{k}^{*}=\left[R_{\alpha_{k}}\right]\left(\left[R_{\theta_{k}}\right]\left(\mathbf{p}_{1}^{*}-\mathbf{a}_{0}\right)+\mathbf{a}_{0}-\mathbf{a}_{n k}\right)+\mathbf{a}_{n k}, k=6,7 \ldots 9 \\
& \mathbf{q}_{k}^{*}=\left[\begin{array}{l}
\left.R_{\alpha_{k}}\right]\left(\left[R_{\theta_{k}}\right]\left(\mathbf{q}_{1}^{*}-\mathbf{a}_{0}\right)+\mathbf{a}_{0}-\mathbf{a}_{n k}\right)+\mathbf{a}_{n k}, k=6,7 \ldots 9 \\
\left\{\begin{array}{c}
\theta_{j}>\theta_{j-1} \\
\theta_{9}<2 \pi
\end{array} j=3,4 \ldots 9\right.
\end{array}\right.
\end{aligned}
$$

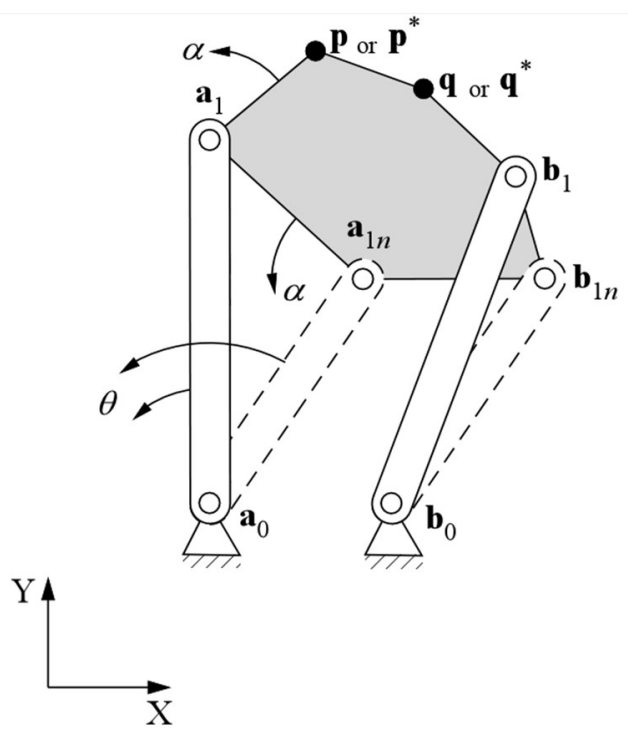

Fig. 3 Adjustable planar four-bar linkage dimensions and angular displacement variables.

\section{Planar four-bar centrode generation}

Geometrically speaking, the instant center of interest in this work is the point of intersection between lines that pass through the crank and follower links (point $\mathbf{X}$ in Figure 2). To repeat, a centrode (a locus of instant centers) is produced over the motion of the linkage. Equations (17) and (18) calculate the fixed and moving centrodes of a planar four-bar linkage respectively. These equations were originally formulated for the spatial four-bar RRSS linkage (Shen et al., 2011). But they can accommodate the planar four-bar linkage by constraining the equation terms to motion in the $x-y$ plane. A detailed formulation procedure for the instant center equation is provided in the noted reference (Shen et al., 2011). As presented, both equations apply to the moving pivot adjustments $\mathbf{a}$ and $\mathbf{b}$. To calculate centrodes for the moving pivot adjustments $\mathbf{a}_{n}$ and $\mathbf{b}_{n}$, moving pivots $\mathbf{a}$ and $\mathbf{b}$ should be replaced with $\mathbf{a}_{n}$ and $\mathbf{b}_{n}$ respectively in Equations (17) and (18). The vector $\hat{\mathbf{u}}$ in both equations is a unit vector orthogonal to the $\mathrm{x}-\mathrm{y}$ plane.

$$
\mathbf{X}_{\text {fixed }}=\mathbf{b}_{0}+\frac{\left[\left(\mathbf{a}-\mathbf{a}_{0}\right) \times \hat{\mathbf{u}}\right] \cdot\left(\mathbf{b}_{0}-\mathbf{a}_{0}\right)}{\left[\left(\mathbf{a}-\mathbf{a}_{0}\right) \times \hat{\mathbf{u}}\right] \cdot\left(\mathbf{b}_{0}-\mathbf{b}\right)}\left(\mathbf{b}-\mathbf{b}_{0}\right)
$$




$$
\mathbf{X}_{m o v i n g}=\mathbf{b}+\frac{\left[\left(\mathbf{a}_{0}-\mathbf{a}\right) \times \hat{\mathbf{u}}\right] \cdot(\mathbf{b}-\mathbf{a})}{\left[\left(\mathbf{a}_{0}-\mathbf{a}\right) \times \hat{\mathbf{u}}\right] \cdot\left(\mathbf{b}-\mathbf{b}_{0}\right)}\left(\mathbf{b}_{0}-\mathbf{b}\right)
$$

\section{Example}

\subsection{Motion generation}

Table 1 includes the planar Cartesian coordinates for two groups of five digging bucket positions (Figure 4). The first group is the position sequence 1-2-3-4-5 and the second group is the sequence 1-6-7-8-9. The dimensions of an adjustable planar four-bar motion generator were calculated to achieve these bucket positions.

As noted in Section 2, the optimization model presented was codified in the commercial mathematical analysis software Matlab and solved using the interior point algorithm. To calculate the unknown optimization model variables, initial values for these variables must be specified first. Table 2 includes the (arbitrary) initial specified values for the dimensions and displacement angles of the adjustable planar four-bar linkage. After specifying the initial values, the interior point algorithm was then executed in Matlab to calculate the final values of the optimization model variables. Table 2 includes the final calculated values for the dimensions and displacement angles of the adjustable planar four-bar linkage. The digging bucket position sequence 1-2-3-4-5 is achieved with the follower link moving pivots $\mathbf{a}_{1}$ and $\mathbf{b}_{1}$. The sequence 1-6-7-8-9 is achieved with the moving pivots $\mathbf{a}_{1 n}$ and $\mathbf{b}_{1 n}$. Table 2 also includes the resulting objective function value (Equation 1) with the calculated values as well as the number of solution iterations and the elapsed clock time for the interior point algorithm to reach the calculated values. Table 3 includes the digging bucket position coordinates achieved by the calculated adjustable planar four-bar motion generator (Figure 5).

Table 1. Prescribed digging bucket position coordinates (unitless).

\begin{tabular}{c|c|c}
\hline \hline Position & $\mathbf{p}$ & $\mathbf{q}$ \\
\hline 1 & $-1.5655,-0.9643$ & $0.1598,-0.1598$ \\
2 & $-1.1364,-3.2642$ & $0.3533,-2.0790$ \\
3 & $-0.0691,-5.2861$ & $1.0886,-3.7749$ \\
4 & $2.5315,-7.8088$ & $2.8767,-5.9367$ \\
5 & $3.6668,-8.4132$ & $3.6685,-6.5096$ \\
6 & $4.6133,-8.3640$ & $4.4952,-6.4640$ \\
7 & $5.7566,-8.5157$ & $5.3397,-6.6583$ \\
8 & $6.9312,-8.5020$ & $6.2095,-6.7405$ \\
9 & $8.1250,-8.3153$ & $7.1006,-6.7108$ \\
\hline
\end{tabular}




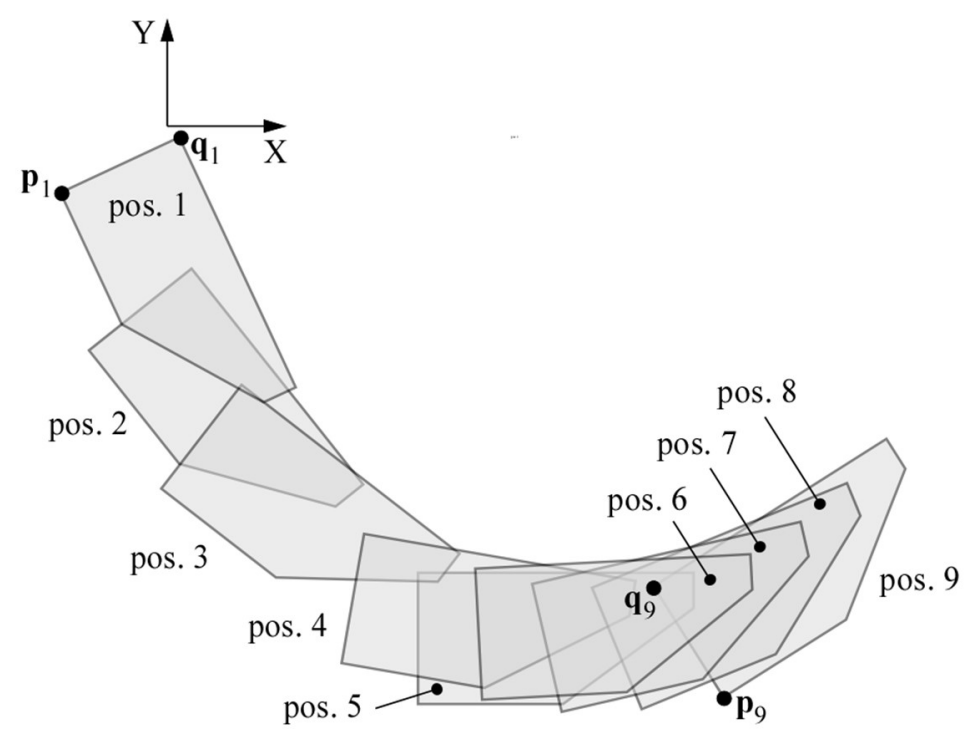

Fig. 4 Prescribed digging bucket positions.

Table 2. Initial and calculated adjustable planar four-bar motion generator values.

\begin{tabular}{c|c|c}
\hline \hline Variable & Initial Values & Calculated Values \\
\hline$a_{0 x}, a_{0 y}$ & $10,-1$ & $7.0229,-2.3279$ \\
$a_{1 x}, a_{1 y}$ & $1,-5$ & $-6.7293,-24.1973$ \\
$a_{1 n x}, a_{1 n y}$ & $1,-5$ & $-7.5886,-22.2514$ \\
$b_{0 x}, b_{0 y}$ & $10,-1$ & $8.1235,-0.2474$ \\
$b_{1 x}, b_{1 y}$ & $1,-1$ & $-18.2572,7.7185$ \\
$b_{1 n x}, b_{1 n y}$ & $-10,10$ & $-17.9928,22.1964$ \\
$\theta_{2} \ldots \theta_{9}$ & $10^{\circ} \ldots 10^{\circ}$ & $15.3094^{\circ}, 31.1861^{\circ}, 58.4474^{\circ}, 68.6747^{\circ}, 73.8886^{\circ}, 83.6346^{\circ}$, \\
& & $93.9708^{\circ}, 105.0289^{\circ}$ \\
$\alpha_{2} \ldots \alpha_{9}$ & $10^{\circ} \ldots 10^{\circ}$ & $-1.2548^{\circ},-2.4179^{\circ},-3.8961^{\circ},-4.2284^{\circ},-5.7983^{\circ},-6.2853^{\circ}$, \\
\end{tabular}




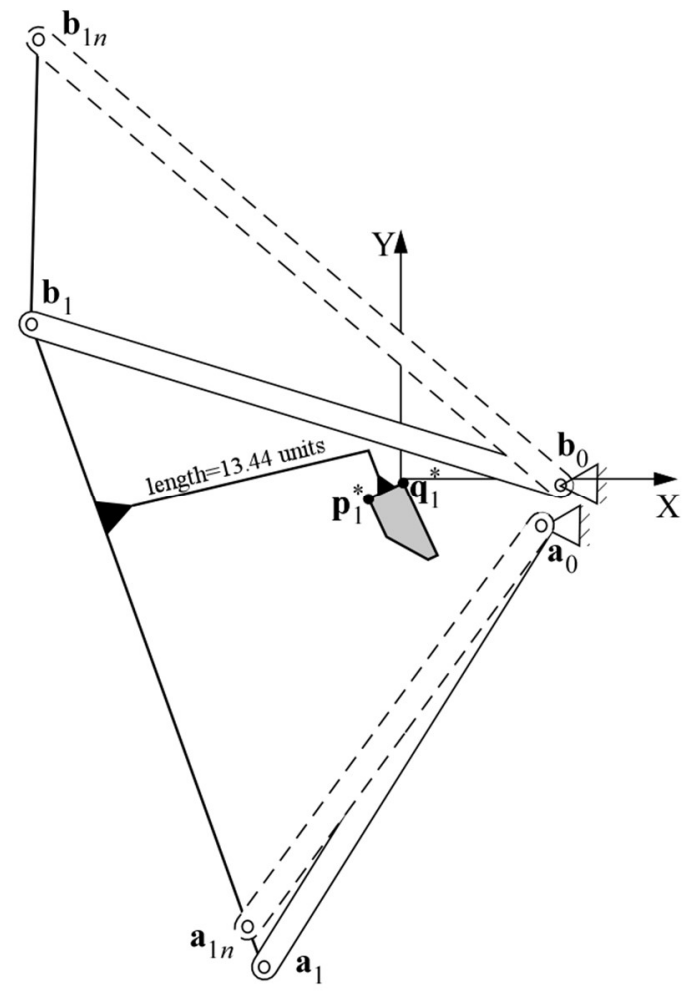

Fig. 5 Calculated adjustable planar four-bar motion generator.

Table 3. Digging bucket position coordinates achieved by calculated planar four-bar motion generator.

\begin{tabular}{c|c|c}
\hline \hline Position & $\mathbf{p}^{*}$ & $\mathbf{q}^{*}$ \\
\hline 1 & $-1.5655,-0.9643$ & $0.1598,-0.1598$ \\
2 & $-1.0997,-3.2607$ & $0.3786,-2.0613$ \\
3 & $-0.0726,-5.3075$ & $1.0526,-3.7720$ \\
4 & $2.5307,-7.8103$ & $2.8758,-5.9382$ \\
5 & $3.6616,-8.4102$ & $3.6801,-6.5067$ \\
6 & $4.6075,-8.3626$ & $4.5049,-6.4617$ \\
7 & $5.7525,-8.5191$ & $5.3454,-6.6595$ \\
8 & $6.9312,-8.5037$ & $6.2091,-6.7424$ \\
9 & $8.1305,-8.3088$ & $7.0910,-6.7140$ \\
\hline
\end{tabular}

\subsection{Centrode generation}

Figure 6 includes the centrodes calculated using Equations (17) and (18) along with calculated adjustable planar motion generator values in Table 2. By rolling the moving centrode over the fixed centrode in Figure 6a, the coordinates for positions 1 through 5 in Table 3 are achieved. The coordinates for positions 1, 6,7,8 and 9 in Table 3 are achieved using the centrodes in Figure 6b. In Figure 7, the centrodes achieving positions 1 and 5 (the initial and final positions in the first group) are illustrated. In Figure 8, the centrodes achieving an intermediate position and position 9 are illustrated. In Figures 7 and 8, the rolling motion of the moving centrode over the fixed centrode between the initial and final positions in each group can be observed. 


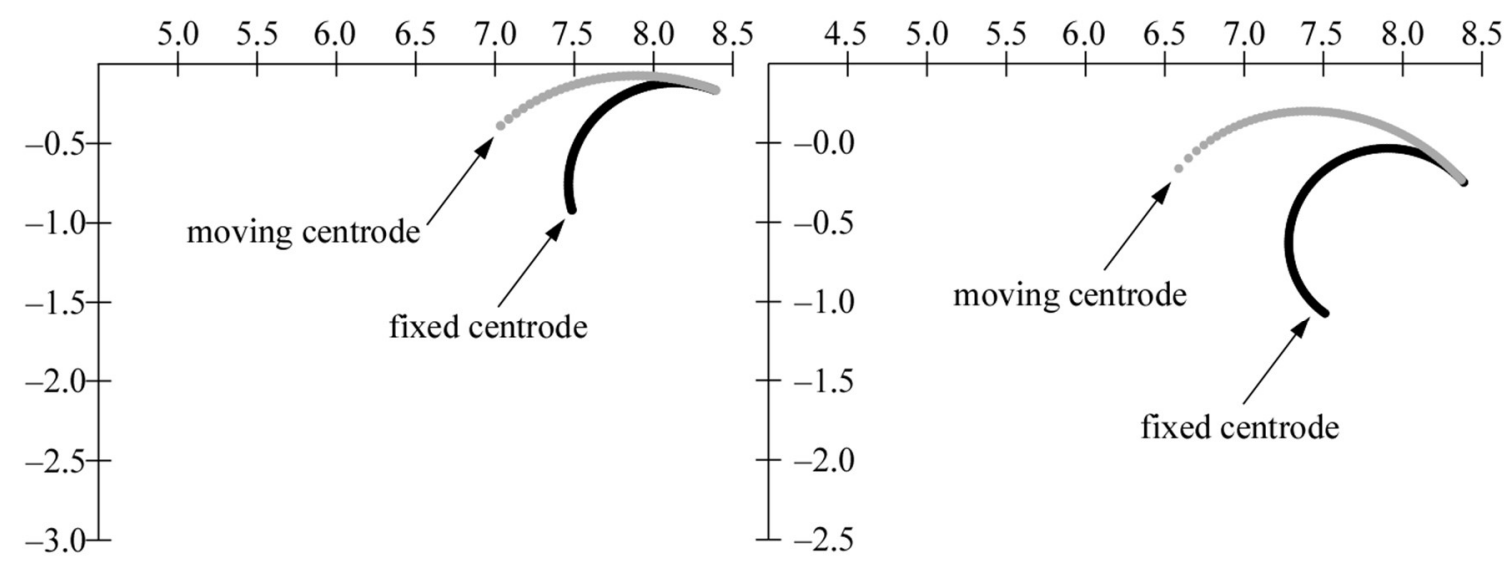

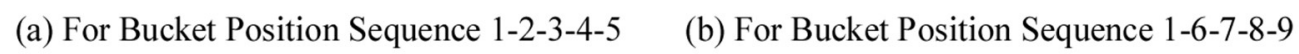

Fig. 6 Calculated fixed and moving centrodes for adjustable planar four-bar motion generator (with unitless axis scales).
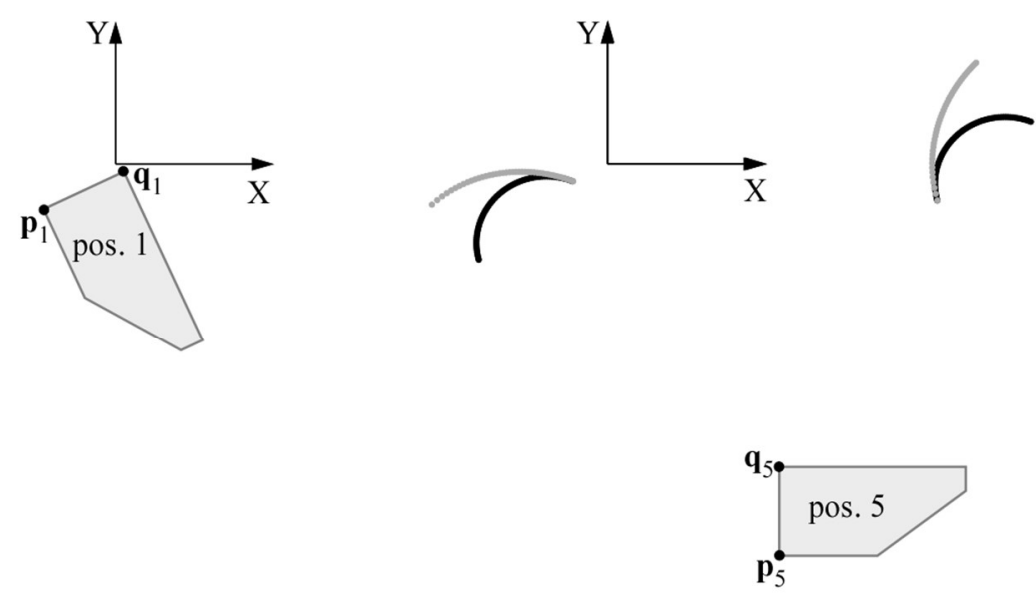
(a) Centrodes at Initial Bucket Position
(b) Centodes at Final Bucket Position

Fig. 7 Centrodes at (a) initial and (b) final bucket positions in bucket position sequence 1-2-3-4-5.
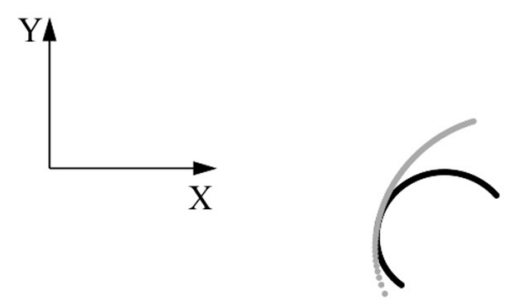

Moving centrode rolled over fixed centrode until bucket matches orientation at pos. 5 .

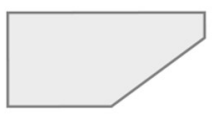

(a) Centrodes at Intermediate Bucket Position
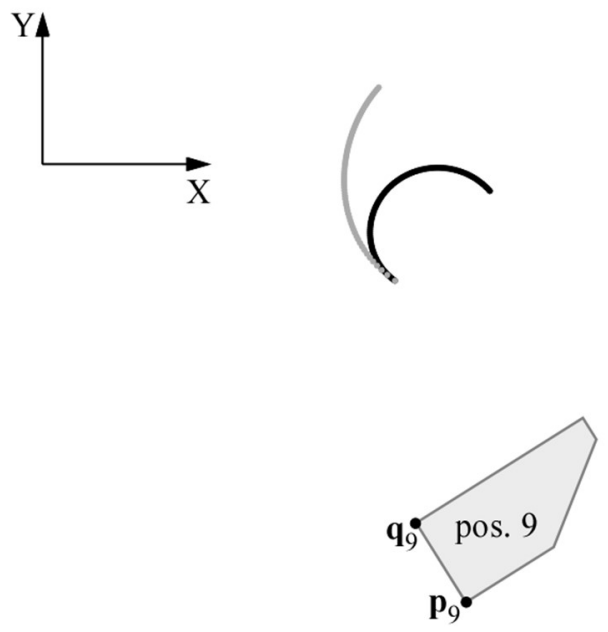

(b) Centodes at Final Bucket Position

Fig. 8 Centrodes at (a) intermediate and (b) final bucket positions in bucket position sequence 1-6-7-8-9. 


\subsection{Cam system design}

The calculated centrodes were incorporated into the design of a cam system. Specifically, the moving centrodes in Figure $7 \mathrm{~b}$ and Figure $8 \mathrm{a}$ were incorporated. In Figure 8a, the moving centrode was rolled over the fixed centrode to reflect a crank rotation of $70.6144^{\circ}$ (between $\theta_{5}$ and $\theta_{6}$ in Table 2). This was done to match the bucket orientation in position 5. Because the rolling motion of the moving centrode over the fixed centrode matches the coupler motion in the corresponding four-bar linkage, it was determined (by a kinematic displacement analysis) that a $70.6144^{\circ} \mathrm{crank}$ rotation is required to achieve the digging bucket position in Figure 8a.

The moving centrodes were included as cam surfaces in a concept dual cam design (Figure 9) for the adjustable motion generator (Figure 5). The cam design also includes pins that will interface slots to keep the moving and fixed centrodes in contact during rolling motion. Because the coupler motion of a four-bar linkage matches the rolling motion of its moving centrode over its fixed centrode, the cam pins in Figure 9 are prescribed coupler points on the calculated linkage and the pin slots in Figure 10 are the resulting coupler curves at the prescribed coupler points. The digging bucket would be connected to the cam component.

In Figure 10, the fixed centrodes are included as cam surfaces in concept side plate components that interface the dual cam. Also included in the plate designs are the corresponding pin slots. Side plate \#1 in Figure 10 interfaces with the front view of the cam in Figure 9 and the side plate \#2 interfaces with the back view of the cam.

Figure 11 includes an exploded view of the concept cam system. To achieve the position sequence 1-2-3-4-5, only side plate \#1 is translated (e.g., via actuator or solenoid) until the fixed-moving centroid pair and pin-slot pair engages. Once engaged, the dual cam can be driven through the position 1-2-3-4-5 sequence. When position 5 is reached, side plate \#2 is translated into engagement with the dual cam and side plate \#1 is retracted out of engagement. Once this occurs, the cam can be driven through the position 6-7-8-9 sequence. Figures 12 and 13 illustrate the concept system, with digging bucket attached, at each of the achieved bucket positions.

Compared to the adjustable four-bar linkage (Figure 5), the cam system has a more compact workspace. Also, while the former can require moving pivot disassembly to achieve both groups of prescribed positions, the latter only requires the side plates to be translated in and out of engagement with the cam to achieve the same result.

The cam and slot profiles in the concept cam system are unique to the digging bucket positions used in planar fourbar motion generation. Different prescribed positions will result in different four-bar motion generator dimensions, centrodes and subsequently, different cam and slot profiles in the cam system.

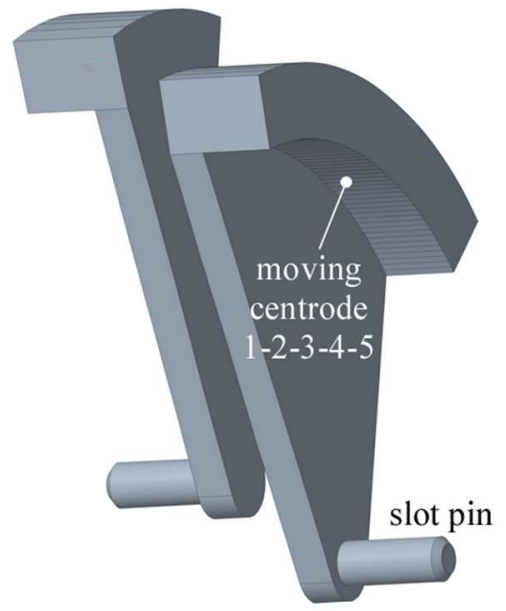

(a) front view

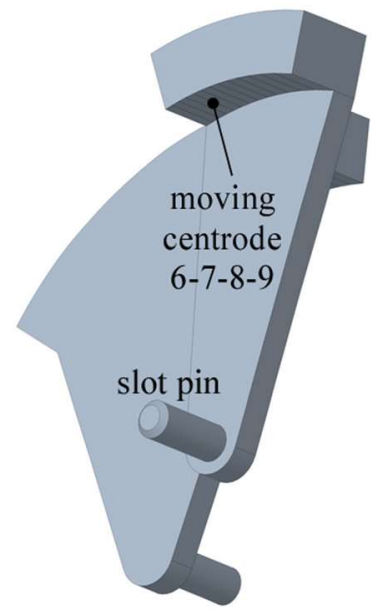

(b) back view

Fig. 9 (a) Front and (b) back views of concept dual cam design for adjustable motion generator. 


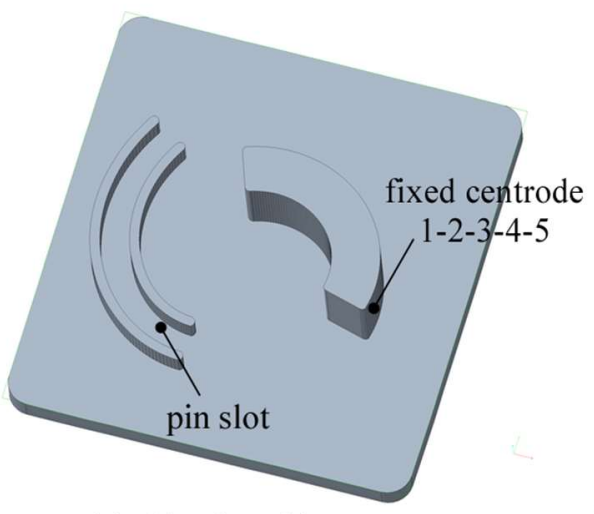

(a) side plate \#1

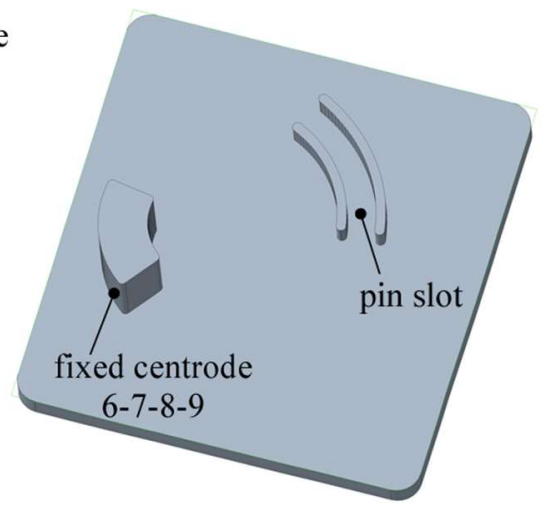

(b) side plate \#2

Fig. 10 Concept designs for (a) side plate \#1 and (b) side plate \#2 for cam system.

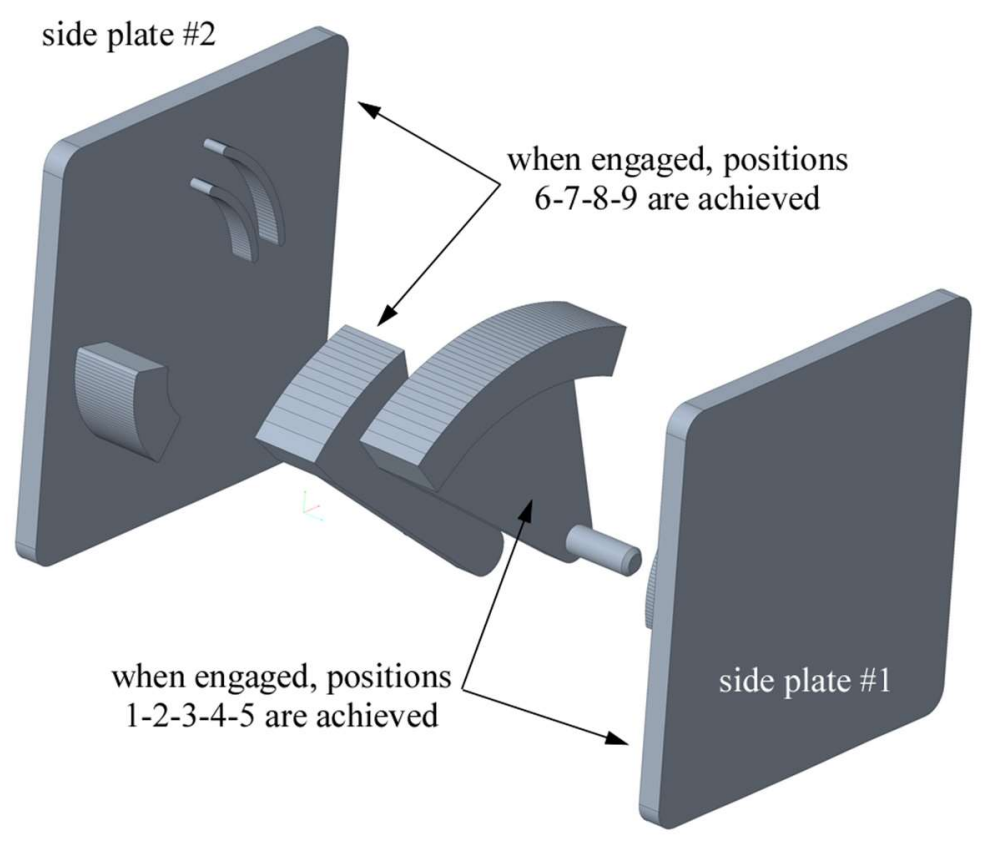

Fig. 11 Exploded view of concept system. 


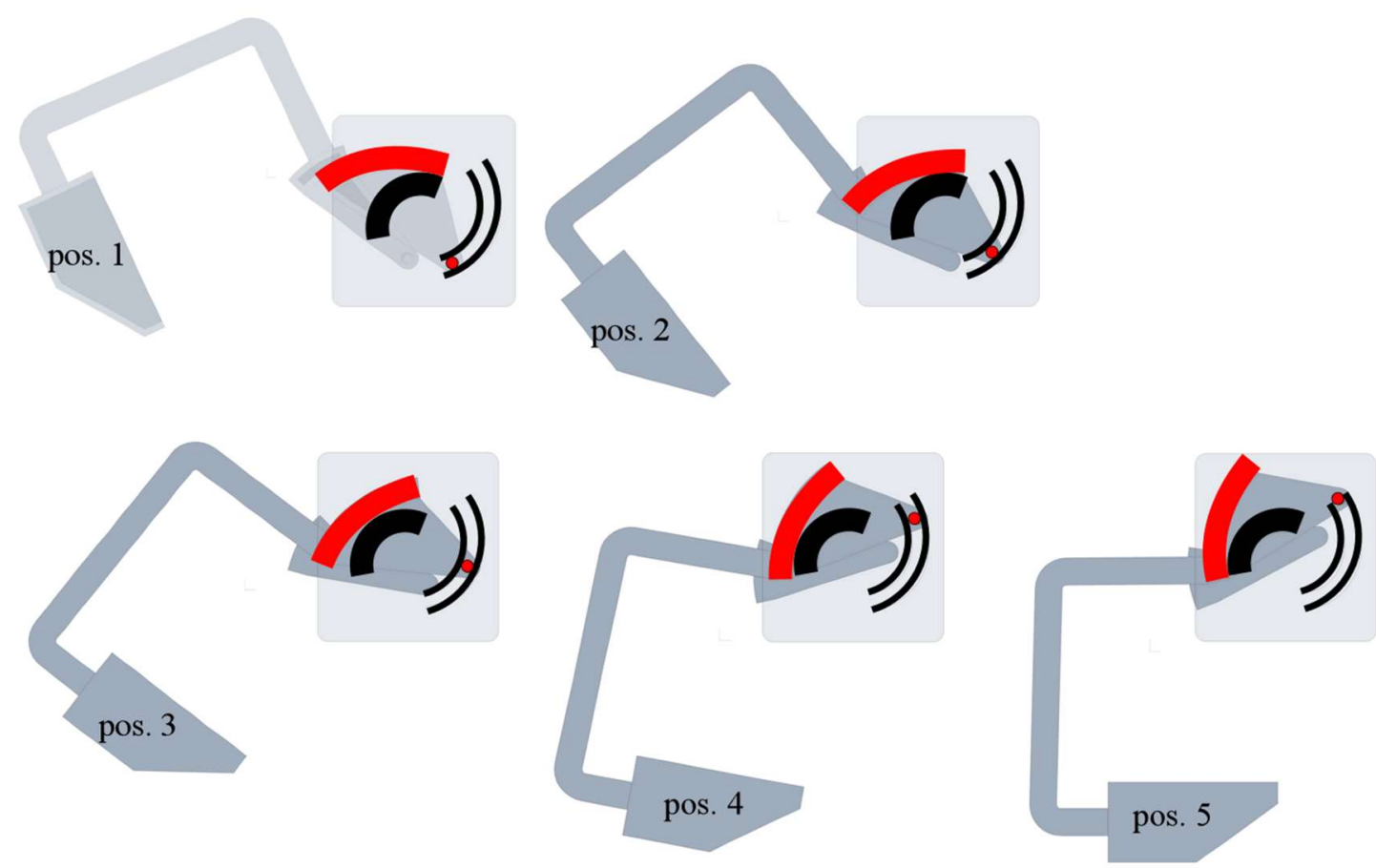

\section{Legend}

Moving Centrode Cam and Slot Pin

Fixed Centrode Cam and Slot

Fig. 12 Concept system (when engaging slide plate \#1) at each achieved digging bucket position.
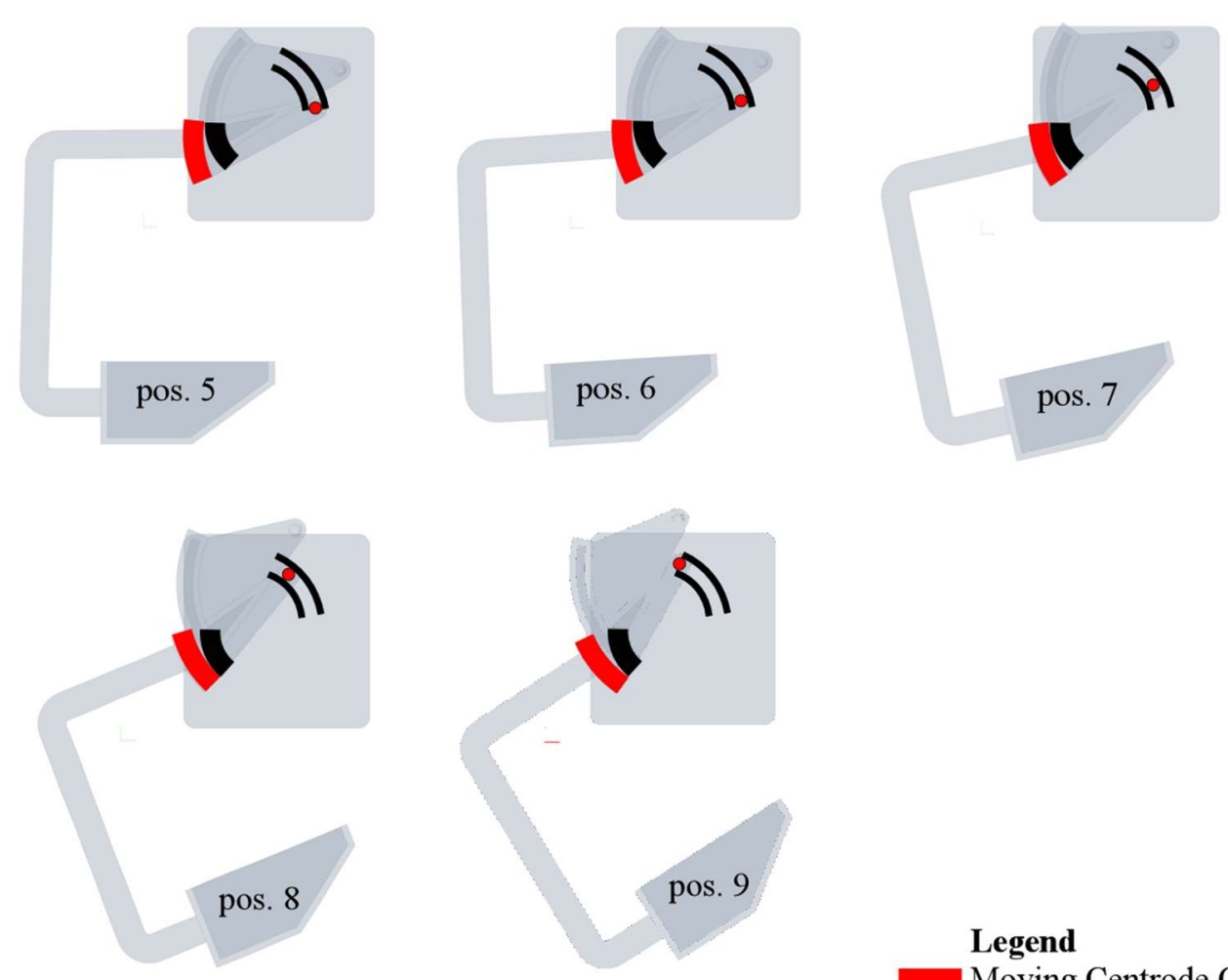

\section{Legend \\ Moving Centrode Cam and Slot Pin \\ Fixed Centrode Cam and Slot}

Fig. 13 Concept system (when engaging slide plate \#2) at each achieved digging bucket position. 


\section{Discussion}

While two groups of five prescribed positions were demonstrated in this work, the optimization model presented in this work can be expanded to accommodate an indefinite number of positions. Motion generation optimization models can be formulated to consider other combinations of crank and follower link adjustments (e.g., constant length moving pivot adjustments or fixed pivot adjustments). For example, replacing $\mathbf{a}_{1 n}$ and $\mathbf{b}_{1 n}$ with $\mathbf{a}_{1}$ and $\mathbf{b}_{1}$ respectively in Equation (2) through (15) produces an optimization model for constant length moving pivot adjustments only. Fixed and moving centrodes can be used as pitch curves for noncircular gears. Circles can also be fitted for the centrode points and used as pitch circles for circular gears (Lee et al, 2020). Gears offer the advantage of higher torque capacity over cam surfaces since the latter relies on friction to maintain slip-free rolling motion.

\section{Conclusion}

This work presents and successfully demonstrates a design method for a cam system for adjustable planar four-bar motion generation. Given two groups of five prescribed digging bucket positions, a defect-free moving pivot adjustable four-bar motion generator was calculated. Its fixed and moving centrodes were calculated and incorporated as cam surfaces in a cam system. The cam system consists of a central rolling dual cam and pin component and two stationary cam-and-slot plate components. The latter components engage the central dual cam component on each side interchangeably to achieve both groups of prescribed positions.

\section{References}

Ahmad, A. and Waldron, K. J., "Synthesis of Adjustable Planar 4-bar Mechanisms," Mechanism and Machine Theory, Vol. 14, No. 6 (1979), pp. 405-411.

Balli, S. S. and Chand, S., "Defects in Link Mechanisms and Solution Rectification," Mechanism and Machine Theory, Vol. 37, No. 9 (2002), pp. 851-876.

Lee, W. and Russell, K., "Developments in Quantitative Dimensional Synthesis (1970-Present): Four-Bar Motion Generation," Inverse Problems in Science and Engineering, Vol. 26, No. 1 (2018), pp. 133-148.

Lee, W., Russell, K., Shen, Q., and Sodhi, R. S., "On Adjustable Spherical Four-bar Motion Generation for Expanded Prescribed Positions," Mechanism and Machine Theory, Vol. 44, No. 1 (2009), pp. 247-254.

Lee, W., Russell, K. and Sodhi, R.S., "On Transfermoral Prosthetic Knee Design for Natural Human Knee Motion," Recent Patents on Mechanical Engineering, Vol. 13, No. 1 (2020), pp. 49-59.

Nocedal, J. and Wright, S.J., Numerical Optimization (2006), Chapter 19, Springer, New York, New York.

Russell, K., Shen, Q. and Sodhi, R.S., Kinematics and Dynamics of Mechanical Systems: Implementation in Matlab and SimMechanics (2019), Chapter 4, CRC Press, New York, New York.

Russell, K. and Sodhi, R. S., "Kinematic Synthesis of Adjustable RRSS Mechanisms for Multi-phase Motion Generation," Mechanism and Machine Theory, Vol. 36, No. 8 (2001), pp. 939-952.

Russell, K. and Sodhi, R. S., "Kinematic Synthesis of RRSS Mechanisms for Multi-phase Motion Generation with Tolerances," Mechanism and Machine Theory, Vol. 37, No. 3 (2002), pp. 279-294.

Russell, K. and Sodhi, R. S., "Kinematic Synthesis of RRSC mechanisms for Multi-phase Finite and Multiply-separated Positions," Transactions of the Canadian Society for Mechanical Engineering, Vol. 26, No. 3 (2002), pp. 263-280.

Q. Shen, K. Russell, W. Lee and R. S. Sodhi, "On Cam System Design to Replicate Spatial Four-Bar Mechanism Coupler Motion," Inverse Problems in Science and Engineering, Vol. 19, No. 2 (2011), pp. 251-265.

Shen, Q., Lee, W. and Russell, K., "On Adjustable Planar Four-bar Motion Generation with Order, Branch and Circuit Defect Rectification," Journal of Mechanisms and Robotics, Vol. 7, No. 3 (2015), pp. 034501.

Sodhi, R. S. and Russell, K., "Kinematic Synthesis of Planar Four-bar Mechanisms for Multi-phase Motion Generation with Tolerances," Mechanics Based Design of Structures and Machines, Vol. 32, No. 2 (2004), pp. 215-233.

Suh, C. H. and Radcliffe, C. W., Kinematics and Mechanism Design (1978), John Wiley and Sons Inc., New York, New York.

Venkataramanujam, V. and Larochelle, P. M., "Design and Development of Planar Reconfigurable Motion Generators," Mechanics Based Design of Structures and Machines, Vol. 44, No. 4 (2016), pp. 426-439. 
Wang, S. J. and Sodhi R. S., "Kinematic Synthesis of Adjustable Moving Pivot Four-bar Mechanisms for Multi-phase Motion Generation," Mechanism and Machine Theory, Vol. 31, No. 4 (1996), pp. 459-474.

Wu, T. and Chen, C., "Mathematical Model and its Simulation of Exactly Mechanism Synthesis with Adjustable Link," Applied Mathematics and Computation, Vol. 160, No. 2 (2005), pp. 309-316.

Zhou, H. and Cheung, E. H. M., "Adjustable Four-bar Linkages for Multi-phase Motion Generation," Mechanism and Machine Theory, Vol. 39, No. 3 (2004), pp. 261-279. 